\title{
Visualization of Damage Progress in Solid Oxide Fuel Cells*
}

\author{
Ken-ichi FUKUI* ${ }^{* *}$, Shogo AKASAKI ${ }^{* * *, \dagger}$, Kazuhisa SATO $^{\dagger \dagger}$, \\ Junichiro MIZUSAKI $^{\dagger \dagger}$, Koichi MORIYAMA**, Satoshi KURIHARA ${ }^{* *}$ and \\ Masayuki NUMAO** \\ ** The Institute of Scientific and Industrial Research, Osaka University \\ 8-1 Mihogaoka, Ibaraki, Osaka 567-0047, Japan \\ E-mail: fukui@ai.sanken.osaka-u.ac.jp \\ *** Graduate School of Information Science and Technology, Osaka University \\ 1-5 Yamadaoka, Suita, Osaka 565-0871, Japan \\ $\dagger$ currently at DENSO Corporation \\ 1-1 Showa-cho, Kariya, Aichi 448-8661, Japan \\ $\dagger \dagger$ Institute of Multidisciplinary Research for Advanced Materials, Tohoku University \\ 2-1-1 Katahira, Aoba-ku, Sendai 980-8577 Japan
}

\begin{abstract}
The fuel cell is regarded as a highly efficient, low-pollution power generation system. In particular, Solid Oxide Fuel Cell (SOFC) has a high generation efficiency. However, a crucial issue in putting SOFC to practical use is the establishment of a technique for evaluating the deterioration. We previously developed a technique by which to measure the mechanical damage of SOFC using the Acoustic Emission (AE) method. In the present paper, we applied the kernel Self-Organizing Map (SOM), which is an extended neural network model, to produce a cluster map reflecting the similarity of AE events. The obtained map visualized the change in occurrence patterns of similar AE events, revealing four phases of damage progress. The methodology of the present study provides a common foundation for a comprehensive damage evaluation system and a damage monitoring system.
\end{abstract}

Key words : Solid Oxide Fuel Cells, Damage Evaluation, Acoustic Emission, SelfOrganizing Map

\section{Introduction}

The fuel cell is regarded as a highly efficient, low-pollution power generation system that produces electricity by direct chemical reaction. Solid Oxide Fuel Cells (SOFCs), in particular, have attracted a great deal of attention because they have a power generation efficiency of nearly $70 \%$ when combined with a gas turbine ${ }^{(1)}$. One of the most important issues for the practical use of SOFCs is to ensure the reliability of $0.25 \% / 1,000 \mathrm{~h}$ at 40,000 hours of operation. However, since SOFCs operate at a high temperature $\left(600^{\circ} \mathrm{C}-1,000^{\circ} \mathrm{C}\right)$ and in oxidation-reduction environments, there are concerns that chemical degradation, such as a change in the micro-structure of the materials, attachment of impure substances, and inter-diffusion, and physical degradation, such as crack or delamination ${ }^{(2)-(4)}$. Putting SOFCs to practical use requires the establishment of a technique for evaluating the deterioration of SOFCs in the operating environment. Therefore, we attempt to clarify the fracture mechanism of SOFCs by combining an electrochemical method for the chemical degradation and a non-destructive testing method for the physical degradation.

In the previous study, we succeeded in measuring physical damage on a single SOFC by Japanese Original: Trans. Jpn. Soc. Mech. Eng., Vol.76, No.762, A (2010), pp.223-232 (Received 1 July, 2009) [DOI: 10.1299/jee.6.499]

Copyright (c) 2011 by JSME the acoustic emission (AE) method ${ }^{(5)}$. However, few studies have dealt with such physical degradation compared to chemical degradation. Previously, the details of the fracture mechanism of SOFC are unclear because it is difficult to monitor the internal state of the cells 
under operation. Here, although it is advantageous that the shape of the SOFC can be changed depending on the purpose, the fracture mechanism and its process also change. Therefore, methods of analyzing AE events must be independent of materials, shape, and temperature. In addition, SOFCs consist of several components, and it is possible for a large number of AE events to occur under long-term operation.

Therefore, in the present paper, we developed a visualization methodology as an analysis basis of observed AE events that reflects the similarity of the AE events, i.e., a twodimensional map, so as to preserve, to the greatest extent possible, the similarity among AE events. It is useful to automatically generate clusters of a large number of AE events containing multiple damage types and to visualize these events within a two-dimensional map. Henceforth, we refer to this map as a cluster map. The generation of such a map has the following benefits:

- Comprehensive Evaluation: Similar AE events are assumed to have occurred from a similar damage mechanism. Therefore, a cluster of AE events corresponds to a damage type, i.e., a material and its fracture mode. Moreover, damage progress can be evaluated based on the temporal change of regions of frequently occurring AE events in the cluster map.

- Exploratory Analysis: The user can intuitively understand the entire picture of a large number of observed AE events, and explore particular AE events or high-frequency events.

For the generation of a cluster map, we used the Self-Organizing Map (SOM) ${ }^{(6)}$, which is an artificial neural network learning. The SOM was originally a model of associative memory, but has recently been widely used for visual data mining, for example, in exploratory analysis support of documents ${ }^{(7)}$, for the monitoring of machinery ${ }^{(8)}$, and for application to medical care or economics.

Here, the distribution of the frequency spectrum of AE events contains informative characteristics of the event. A spectrum contains a clear distribution structure. However, the standard SOM uses the square norm (Euclidean distance) as a similarity measure of data points. That is, each discrete frequency spectrum point is treated as an independent variable, and so the distribution structure cannot be captured.

Whereas the present paper proposes the use of Kullback-Leibler (KL) divergence to introduce a distribution structure into a similarity measure of damage events. Since the KL divergence was originally a distance metric developed for probability distributions, we assume a frequency spectrum as a probability distribution, in the same manner that Moreno et al. applied the KL divergence to an image spectrum classification ${ }^{(9)}$. In the present study, the kernel SOM${ }^{(10),(11)}$ was used to introduce the KL divergence to the SOM.

The AE method is widely used for non-destructive testing of structures or materials ${ }^{(12)}$. Few studies have examined the automatic classification of AE events. Rippengill et al. ${ }^{(13)}$ classified AE events of a box-girder bridge by means of a multi-layer neural network. In addition, Godin et al. ${ }^{(14)}$ combined k-means clustering and SOM in order to visualize AE events obtained by tensile testing of a polyester composite. However, the features of the AE events in these studies are based on, e.g., duration, rise time, or energy. These features are not sufficient for revealing the damage type in the case of complex damage, such as damage to SOFCs, as described in a preliminary study ${ }^{(15)}$.

Previously, we validated the clustering accuracy of the kernel SOM based on KL divergence for damage events ${ }^{(16)}$. Since the correspondence between damage type and AE events on SOFCs is unknown, we prepared benchmark datasets of, for example, damage-related sounds, cracks in a wood block, and friction of a box. The benchmark datasets reveal that the proposed KL divergence-based kernel SOM yield the best accuracy among the standard kernel functions and the normal SOM. Therefore, the proposed method is expected to provide a highly accurate map for SOFC damage.

In the present study, we applied the KL divergence-based kernel SOM to an AE event sequence observed from a damage evaluation test on SOFC. We validate the effectiveness of the proposed approach by demonstrating that the cluster map can visualize the change in 
occurrence patterns of similar AE events, revealing four phases of damage progress.

\section{Kernel Method}

\subsection{Overview}

In general, a kernel method extends a linear classification or clustering method to obtain a non-linear classification or clustering method or introduces an appropriate similarity measure to existing methods using a kernel function ${ }^{(17)}$. The representative classifier using a kernel method is a Support Vector Machine (SVM), which has been used successfully in various domains, such as speech recognition, document classification, and bioinfomatics ${ }^{(18)}$. Here, it is important to select or construct a kernel function that adapts to the objective domain. The typical kernel functions are Gaussian, Polynomial, and Sigmoid kernels, whereas the Kullback-Leibler (KL) kernel is used in the present study. Ishigaki and Higuchi ${ }^{(19)}$ revealed that the KL kernel is robust for shifting of the spectrum distribution and applied an SVM with a KL kernel for failure diagnosis of a pressure adjuster providing accurate diagnosis, as compared to using general kernel functions.

Formally, given $N$ inputs $\mathbf{x}_{1}, \cdots, \mathbf{x}_{N} \in \mathbb{R}^{v}$, the kernel function $K: \mathbb{R}^{v} \times \mathbb{R}^{v} \rightarrow \mathbb{R}$ is a function that satisfies the following conditions:

- $\quad$ Symmetry $K\left(\mathbf{x}_{i}, \mathbf{x}_{j}\right)=K\left(\mathbf{x}_{j}, \mathbf{x}_{i}\right)$.

- Positive definite For all inputs $\mathbf{x}_{1}, \cdots, \mathbf{x}_{N}$ and arbitrary real numbers $\alpha_{1}, \cdots, \alpha_{N}, K\left(\mathbf{x}_{i}, \mathbf{x}_{j}\right)$ satisfies $\Sigma_{i} \Sigma_{j} \alpha_{i} \alpha_{j} K\left(\mathbf{x}_{i}, \mathbf{x}_{j}\right)>0$.

Under these conditions, the existence of a mapping function $\phi: \mathbb{R}^{v} \rightarrow \mathbb{H}$, which maps the data to a different space from the original space, is proven (Mercer's theorem). The kernel function is then defined by:

$$
K\left(\mathbf{x}_{i}, \mathbf{x}_{j}\right)=<\phi\left(\mathbf{x}_{i}\right), \phi\left(\mathbf{x}_{j}\right)>,
$$

where $<,>$ represents an inner product. Here, since only the inner products of data points appear within algorithms such as SVM and SOM, the algorithms require an inner product of data points. This means that a mapped vector $\phi\left(\mathbf{x}_{i}\right)$ is not necessary to calculate, if the inner product is directly calculated. Using this mathematical property, the kernel method computes within the mapped higher-dimensional space.

\subsection{Kullback-Leibler Kernel}

Let $v$-discrete points of a frequency spectrum be $\mathbf{x}_{i}=\left(x_{i, 1}, \cdots, x_{i, v}\right)$. Then, the KL kernel function is defined as:

$$
\begin{aligned}
& K_{\mathrm{KL}}\left(\mathbf{x}_{i}, \mathbf{x}_{j}\right)=\exp \left(-\beta J S\left(\mathbf{x}_{i}, \mathbf{x}_{j}\right)\right), \\
& J S\left(\mathbf{x}_{i}, \mathbf{x}_{j}\right)=K L\left(\mathbf{x}_{i}, \mathbf{x}_{j}\right)+K L\left(\mathbf{x}_{j}, \mathbf{x}_{i}\right)=\sum_{k=1}^{v}\left\{x_{i, k} \log \frac{x_{i, k}}{x_{j, k}}+x_{j, k} \log \frac{x_{j, k}}{x_{i, k}}\right\},
\end{aligned}
$$

where $K L\left(\mathbf{x}_{i}, \mathbf{x}_{j}\right)$ is the Kullback-Leibler divergence, which is the distance between probability distributions, $J S\left(\mathbf{x}_{i}, \mathbf{x}_{j}\right)$ denotes the Jensen-Shannon divergence, which symmetrizes the KL divergence, and $\beta>0$ is a scaling parameter. Note that the spectra must be normalized as $\sum_{k} x_{i, k}=1$, because the KL divergence was originally developed for a probability distribution. When applied to AE data, the KL kernel measures depending not on the power of the spectrum, but rather on the relative distribution of the spectrum.

\subsection{Difference between the L2-norm and the KL kernel}

This section describes the difference between the L2-norm, on which the standard SOM is based, and the KL kernel. Here, L2-norm $D$ between two frequency spectra $\mathbf{x}_{i}$ and $\mathbf{x}_{j}=\mathbf{x}_{i}+\Delta \mathbf{x}$ is given by:

$$
D\left(\mathbf{x}_{i}, \mathbf{x}_{j}\right)=\sum_{k=1}^{v}\left(x_{i, k}-x_{j, k}\right)^{2}=\sum_{k=1}^{v}\left(x_{i, k}-\left(x_{i, k}+\Delta x_{k}\right)\right)^{2}=\sum_{k=1}^{v} \Delta x_{k}^{2} .
$$




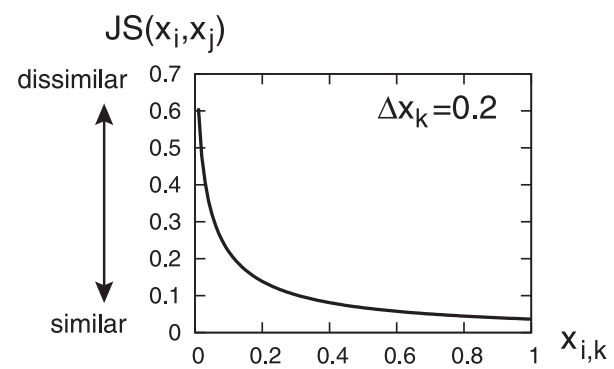

Fig. 1 The function of Jensen-Shannon divergence for a variable of frequency spectrum $x_{i, k}$ when the difference of values of two frequency spectra $\Delta_{k}=0.2$.

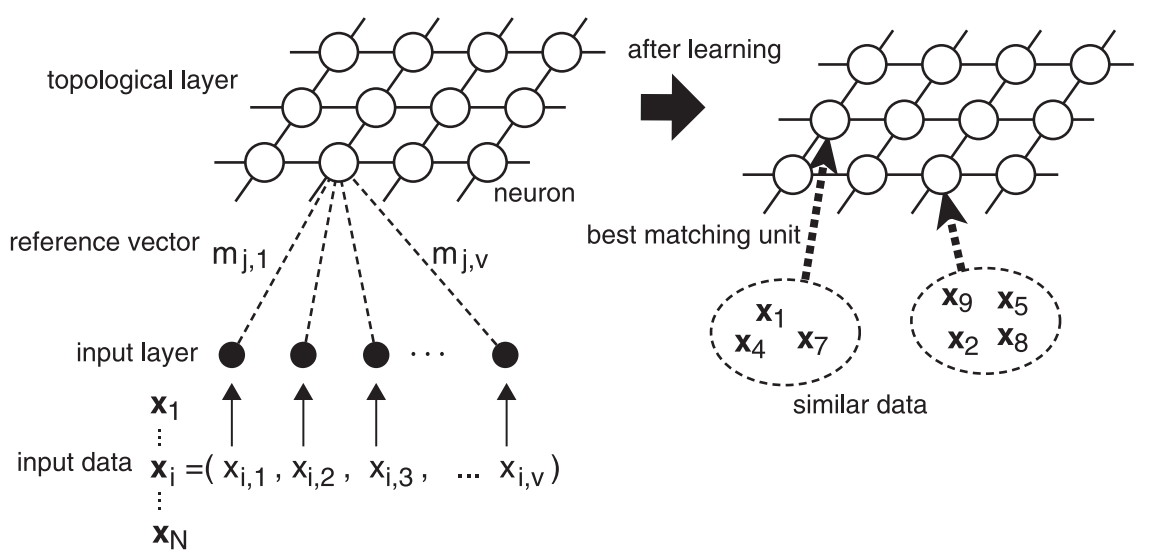

Fig. 2 Concept of the SOM. The SOM learns similar data so as to correspond to neighbors in the neuron topology.

Whereas the component of KL kernel $J S$ is given by:

$$
\begin{aligned}
J S\left(\mathbf{x}_{i}, \mathbf{x}_{j}\right) & =\sum_{k=1}^{v}\left(x_{j, k}-x_{i, k}\right) \log \frac{x_{j, k}}{x_{i, k}} \\
& =\sum_{k=1}^{v} \Delta x_{k} \log \left(\frac{x_{i, k}+\Delta x_{k}}{x_{i, k}}\right) \\
& =\sum_{k=1}^{v} \Delta x_{k} \log \left(1+\frac{\Delta x_{k}}{x_{i, k}}\right) .
\end{aligned}
$$

The L2-norm uniformly measures the difference $\Delta \mathbf{x}$ over the spectra, whereas the KL kernel takes into account the ratio of $x_{i, k}$ to $\Delta x_{k}$. Fig. 1 shows the function of Jensen-Shannon divergence where the difference of two frequency spectra are evaluated. When the power of a certain frequency $x_{i, k}$ is larger, the KL kernel underestimates $\Delta x_{k}$. In contrast, the smaller $x_{i, k}$, the greater the effect of $\Delta x_{k}$. That is, the KL kernel evaluates the difference around a low power frequency rather than that near a spectrum peak. Since a frequency spectrum has sharp peaks, the KL kernel evaluates the spectrum distribution in the sense that the differences near the spectrum peaks are underestimated to some degree.

\subsection{Kernel SOM}

This section describes the extended kernel Self-Organizing Map (SOM) and the basic concept of the original SOM proposed by Kohonen ${ }^{(6)}$. The learning of SOM involves updating the codebook vector, i.e., the connectivity weights between input variables and output neurons, so as to preserve similar data in the same neuron or a neighbor neuron (Fig. 2). Finally, neighbor data are mapped to a low-dimensional representation of the topology space.

The basic concept of the kernel SOM is the same as that of the SOM. However, in the kernel SOM, the reference vector is updated in an indirect manner because the reference vector in the mapped space $\mathbb{H}$ cannot be calculated. 
Suppose that $N$ input data $\left\{\mathbf{x}_{1}, \cdots, \mathbf{x}_{N}\right\} \in \mathbb{R}^{v}$ are given, where $\mathbf{x}_{i}=\left(x_{i, 1}, \cdots, x_{i, v}\right)$ is a $v$-dimensional datum. Let $M$ neurons of the reference vectors in the mapped space be $\left\{\mathbf{m}_{1}, \cdots, \mathbf{m}_{M}\right\} \in \mathbb{H}$. In addition, let the position of $M$ neurons in the topological layer be $\mathbf{r}_{j}=\left(x_{j}, y_{j}\right): j=1, \cdots, M$. Here, the number of neurons and the layout of the topological layer must be pre-defined, and a regular or hexagonal grid is normally used. The kernel SOM then tries to minimize the following objective function by updating reference vectors.

$$
L_{\mathrm{ksom}}=\sum_{i=1}^{M} \sum_{\mathbf{x}_{n} \in C_{i}} \sum_{j=1}^{M} h_{i, j}\left\|\phi\left(\mathbf{x}_{n}\right)-\mathbf{m}_{j}\right\|^{2},
$$

where $C_{i}$ indicates a set of data points for which the winner neuron, which is the nearest neuron, is the $i^{t h}$ neuron. That is, $C_{i}=\left\{\mathbf{x}_{n} \mid c(n)=i\right\}$, where $c(n)$ is the index of the winner neuron to a data point $\mathbf{x}_{n}$. In addition, $h_{i, j}$ is a neighborhood function defined on the topology space, and the following Gaussian function is normally used:

$$
h_{i, j}=\exp \left(-\frac{\left\|\mathbf{r}_{i}-\mathbf{r}_{j}\right\|^{2}}{2 \sigma^{2}}\right)
$$

where $\sigma$ is a parameter representing the effect of neighbors. By this neighborhood function, the similarity among data points is preserved on the topology space.

Then, by replacing $\mathbf{x}$ in the updating formula of a reference vector in the standard batch type $\mathrm{SOM}^{(6)}$ by a mapped $\phi(\mathbf{x})$, the following updating formula can be obtained:

$$
\mathbf{m}_{i}(t+1):=\gamma \sum_{n} h_{c(n), i} \phi\left(\mathbf{x}_{n}\right)
$$

where $t$ is an iteration step, and $\gamma$ is a regularization term $\gamma=1 / \Sigma_{n} h_{c(n), i}$. However, since $\phi\left(\mathbf{x}_{n}\right)$ cannot be calculated, the $i^{\text {th }}$ reference vector is updated using the dissimilarity to all data points $\forall n d_{i, n}$, as follows:

$$
\begin{aligned}
& d_{i, n}(t+1) \equiv\left\|\phi\left(\mathbf{x}_{n}\right)-\mathbf{m}_{i}(t+1)\right\|^{2} \\
& =K\left(\mathbf{x}_{n}, \mathbf{x}_{n}\right)-2 \gamma \sum_{j} h_{c(j), i} K\left(\mathbf{x}_{n}, \mathbf{x}_{j}\right)+\gamma^{2} \sum_{k} \sum_{l} h_{c(k), i} h_{c(l), i} K\left(\mathbf{x}_{k}, \mathbf{x}_{l}\right) .
\end{aligned}
$$

The following describes the algorithm of the batch type kernel SOM.

\section{Kernel SOM algorithm}

Step 1. Initialize all dissimilarity between reference vectors and data points $\forall i, n d_{i, n}$ randomly and set the iteration step as $t=1$.

Step 2. Search the best matching units $\{c(1), \cdots, c(N)\}$ for all inputs by the nearest neuron:

$$
c(n)=\arg \min _{i=1, \cdots, M} d_{i, n},
$$

Step 3. Exit if the best matching units $\{c(1), \cdots, c(N)\}$ were not changed or the iteration reached $t=t_{\text {max }}$.

Step 4. Update the dissimilarity of each reference vector to all inputs $\forall n d_{i, n}$ by Eq. (9). Step 5. Decrease the neighborhood radius $\sigma$ and increase the iteration counter $t \rightarrow t+1$. Then, return to Step 2.

\section{SOFC Damage Test and Preprocessing}

\subsection{SOFC damage test condition}

Figure 3 shows a schematic diagram of the SOFC performance test apparatus. In the present study, a single cell was used, and a sintered $\left(\mathrm{CeO}_{2}\right)_{0.8}\left(\mathrm{SmO}_{1.5}\right)_{0.2}(20 \mathrm{SDC})$ disk having a diameter of $16 \mathrm{~mm}$ and a thickness of $1 \mathrm{~mm}$ was used as electrolyte. A cermet of $\mathrm{NiO}-20 \mathrm{SDC}$ (weight ratio of 60:40) was used as the anode, and $\mathrm{La}_{0.6} \mathrm{Sr}_{0.4} \mathrm{Co}_{0.2} \mathrm{Fe}_{0.8} \mathrm{O}_{3}$ (LSCF) was used as the cathode, where both diameters are $10 \mathrm{~mm}$ and the thicknesses of the anode and cathode are $30 \mathrm{~mm}$ and $15 \mathrm{~mm}$, respectively. The seal between the outer $\mathrm{Al}_{2} \mathrm{O}_{3}$ tubes and the cell was achieved by melting a soda glass ring of $0.5 \mathrm{~mm}$ in thickness. 


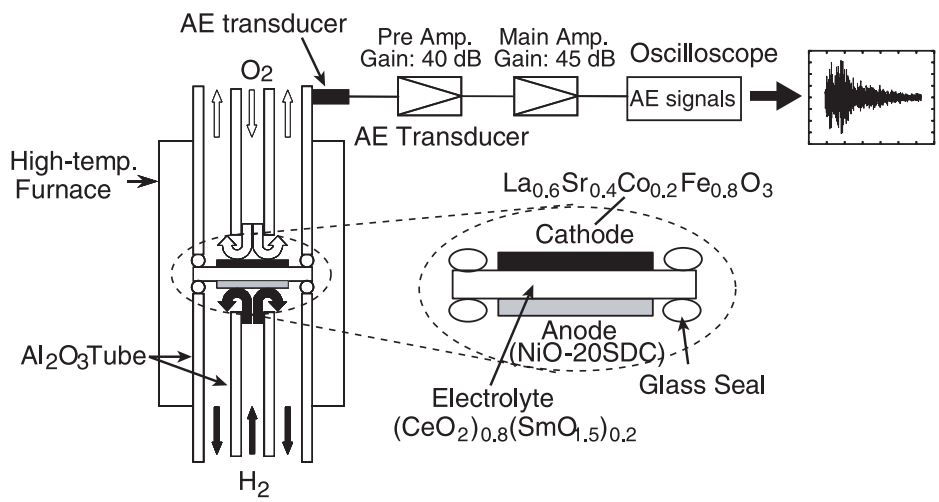

Fig. 3 SOFC damage test and AE measurement apparatus.

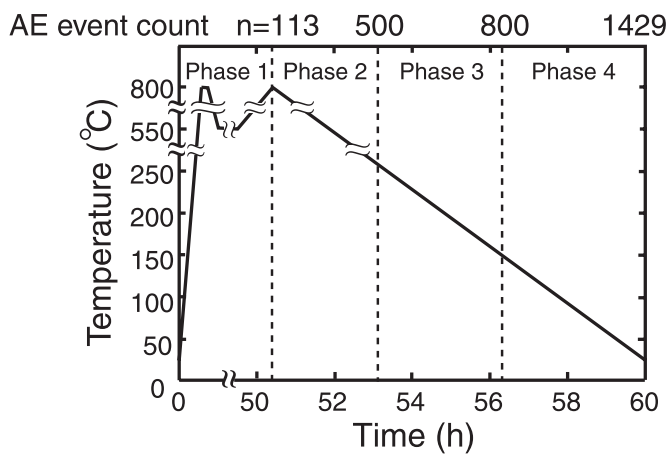

Fig. 4 Correspondence between AE occurrence process phase and temperature history.

The test section was initially heated to $800{ }^{\circ} \mathrm{C}$ at a rate of $100{ }^{\circ} \mathrm{C} / \mathrm{h}$ and was maintained at this temperature for one hour under an atmospheric air environment in order to melt the soda glass ring and form the glass seal (Fig. 4). After melting, the soda glass was then cooled to $550{ }^{\circ} \mathrm{C}$ at rate of $100{ }^{\circ} \mathrm{C} / \mathrm{h}$ and was maintained at this temperature for one hour. At this stage, $\mathrm{H}_{2}$ and $\mathrm{O}_{2}$ gases were introduced to the test section while the temperature was maintained at $550{ }^{\circ} \mathrm{C}$. The temperature was then increased monotonically to $800{ }^{\circ} \mathrm{C}$ and then cooled to room temperature at rate of $100{ }^{\circ} \mathrm{C} / \mathrm{h}$ in order to examine the fracture progress under a heating cycle.

Acoustic emission measurement is performed using a wideband piezoelectric transducer (PAC UT-1000). The AE transducer was attached to the outer $\mathrm{Al}_{2} \mathrm{O}_{3}$ tube away from the heated section, in an area at room temperature. Electric signals from the transducer are amplified using a preamplifier ( $40 \mathrm{~dB})$ and a main amplifier $(45 \mathrm{~dB})$ and then input to an oscilloscope. The sampling rate is $10^{-6}$ seconds. The AE events occurred primarily within the period of temperature decrease.

Photographs of a cathode surface and a cross-section of the cells after the damage test are shown in Fig. 5. We confirmed the existence of vertical cracks in and delamination of the cathode and the electrolyte, as well as cracks in the glass seal. Since the temperature inside the electric furnace is quite high, it is difficult to observe damage in situ. Moreover, since the damage to the SOFC progresses by means of the mechanical mutual influence among the electrode, the electrolyte, and the glass seal, it is difficult to reproduce this damage by a damage test of an individual material. Therefore, the relationship between the AE event and the damage type in the SOFC has not yet been clarified ${ }^{(5)}$.

\subsection{Extraction of $\mathrm{AE}$ events}

First, AE events were extracted from steady noise by the statistical burst extraction $\operatorname{method}^{(20)}$. The noise is assumed to be generated according to a certain constant distribu- 
(a) Cathode Surface

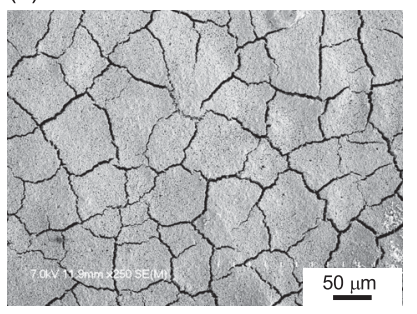

(b) Cross-section

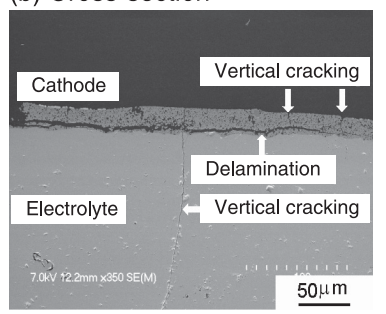

Fig. 5 Photographs of (a) a cathode surface of the cell and (b) a cross-section of the cell after a simulated operating test.

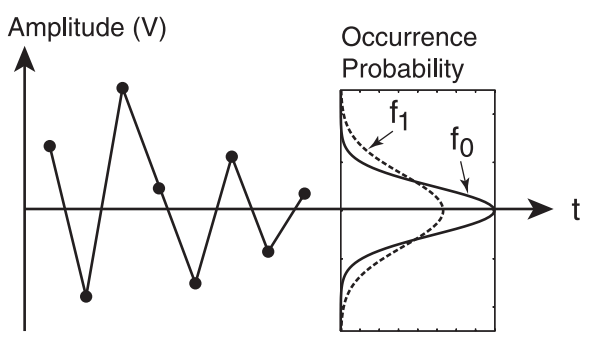

Fig. 6 A diagram of observed AE signals and assumed occurrence probability density functions.

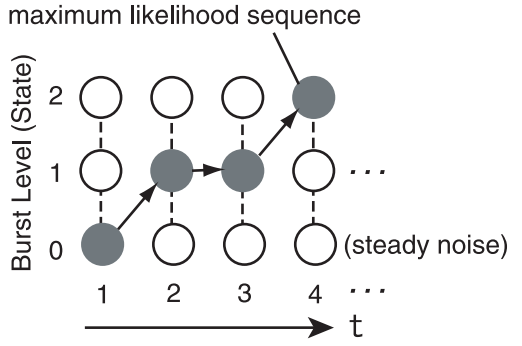

Fig. 7 A state transition for a sequence of AE signals.

tion (steady state), and an AE event is generated by a different distribution. For example, in Fig. 6, AE signals are assumed to be produced by occurrence probability density functions $\mathrm{f}_{0}$ or $\mathrm{f}_{1}$. And the variance of the functions corresponds to the state or burst level that represents the degree of AE activity. The burst extraction method estimates the maximum likelihood state sequence to the observed AE signals (Fig. 7). Moreover, the state transition cost is introduced in order to obtain continued burst periods. Although this method can extract the arbitrary length of an AE event based on the amount of activity of the signals, it is insensitive to the single high-amplitude spike within the noise.

Let $z_{t}\left(t=1, \cdots, t_{\text {end }}\right)$ be an $\mathrm{AE}$ value at time $t$, and $\mathrm{AE}$ signals are assumed to be generated from a Gaussian probability density function:

$$
f_{j}\left(z_{t}\right)=\frac{1}{\sqrt{2 \pi} \sigma_{j}} \exp \left\{-\frac{\left(z_{t}-\mu\right)^{2}}{2 \sigma_{j}^{2}}\right\} \quad(j=0, \cdots, L),
$$

where $\mu=\Sigma_{t} z_{t} / t_{\text {end }}$ is the mean for all AE signals, $\sigma_{0}$ (steady state) is the variance of all AE values, and $\sigma_{j}=s^{j} \sigma_{0}(j \geq 1$, burst state). Here, $s>1$ is a parameter that controls the resolution of bust levels. This model assumes that the different burst levels of the AE signals are generated by the different variances of the Gaussian functions (Fig. 6). Let $\operatorname{Cost}_{j}(t)$ be a necessary cost for $z_{t}$ to be state $j$, then the burst extraction algorithm is as follows: 

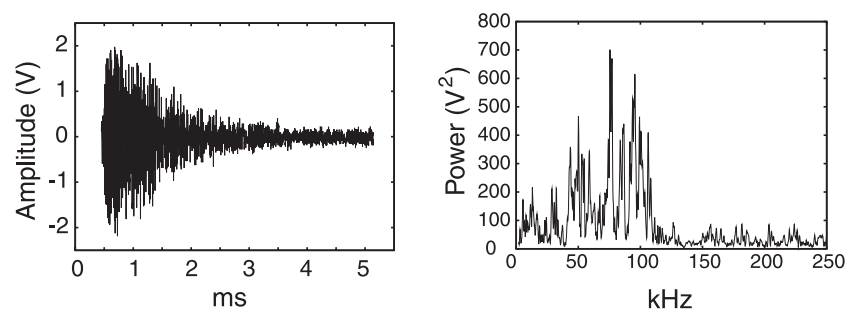

Fig. 8 An example of an extracted AE wave and frequency spectrum.

Burst extraction algorithm

Step 1. Initialize costs at $t=0$ as $\operatorname{Cost}_{j}(0)=0(j=0)$ and $\operatorname{Cost}_{j}(0)=\infty(j \geq 1)$.

Step 2. $t \rightarrow t+1$.

Step 3. Calculate $\operatorname{Cost}_{j}(t)$ for $j=0, \cdots, L$ by the following equation:

$$
\operatorname{Cost}_{j}(t)=-\ln f_{j}\left(z_{t}\right)+\min _{0 \leq l \leq v}\left\{\operatorname{Cost}_{l}(t-1)+\tau(l, j)\right\}
$$

where $j$ is a state at $t$ and $l$ is a state at $t-1$. In addition, $\tau(l, j)$ is the transition cost from state $l$ to $j$ given by

$$
\tau(l, j)=\left\{\begin{aligned}
(j-l) \gamma \ln t_{\text {end }} & \text { if } j>l \\
0 & \text { otherwise, }
\end{aligned}\right.
$$

where $\gamma>0$ is a parameter that controls the effect of transition cost.

Step 4. Continue Steps 2 and 3 until $t=t_{\text {end }}$.

Step 5. Estimate the optimal state sequence that gives the minimum cost using the Viterbi algorithm ${ }^{(21)}$. The Viterbi algorithm traces in the reverse direction from the last signal $t_{\text {end }}$, i.e., the Viterbi algorithm starts from state ${ }^{*}\left(t_{\text {end }}\right)=\arg \min _{0 \leq j \leq L} \operatorname{Cost}_{j}\left(t_{\text {end }}\right)$, and is iterated repeatedly until $t=1$, choosing a previous optimal state as $\operatorname{state}^{*}(t-1)$, which gives the current optimal state $\operatorname{state}^{*}(t)$.

After calculating the optimal burst levels, AE events are obtained by extracting areas where the burst level is greater than $1(j \geq 1)$.

Based on this burst extraction method, we obtained 1,429 AE events through 60 hours of the damage test section, with parameters of $L=5, s=1.5$, and $\gamma=50$. These parameters were tuned manually but are not sensitive to the results.

\subsection{Preprocessing of AE signal}

After extracting AE events, we performed preprocessed in advance of applying the SOM, as follows:

Preprocessing of AE events

Step 1.(Interpolation in the time domain) Let an extracted $\mathrm{AE}$ event be $\mathbf{z}=\left(z_{1}, \cdots, z_{T}\right)$. Note that $T$ varies depending on each AE event. In order to apply FFT, the following linearly interpolation is performed $\mathbf{z}: T \rightarrow 2^{p}\left(2^{p}>>T\right)$.

Step 2.(Frequency analysis) Obtain the frequency power spectrum by FFT.

Step 3. (Frequency filtering) The power spectrum was filtered by $250 \mathrm{kHz}$ for an upper bound because, in this experiment, only low power exists above $250 \mathrm{kHz}$.

Step 4. (Smoothing) Let the discrete points of the power spectrum obtained by Step 3 be $\mathbf{x}=\left(x_{1}, \cdots, x_{V}\right)$. Smoothing is achieved by getting the average of the points together with the adjacent points: $x_{k} \leftarrow\left(x_{k-1}+x_{k}+x_{k+1}\right) / 3(k=2, \cdots, V-1)$. We used three points because peaks sometimes disappeared when five points were used.

Step 5. (Interpolation in the spectrum domain) For the smoothed power spectrum $\mathbf{x}$ obtained in Step 4, linearly interpolate $\mathbf{x}: V \rightarrow v$ in order to apply the SOM that requires a fixed-length input vector. In this experiment, we set $v=4,000$. 


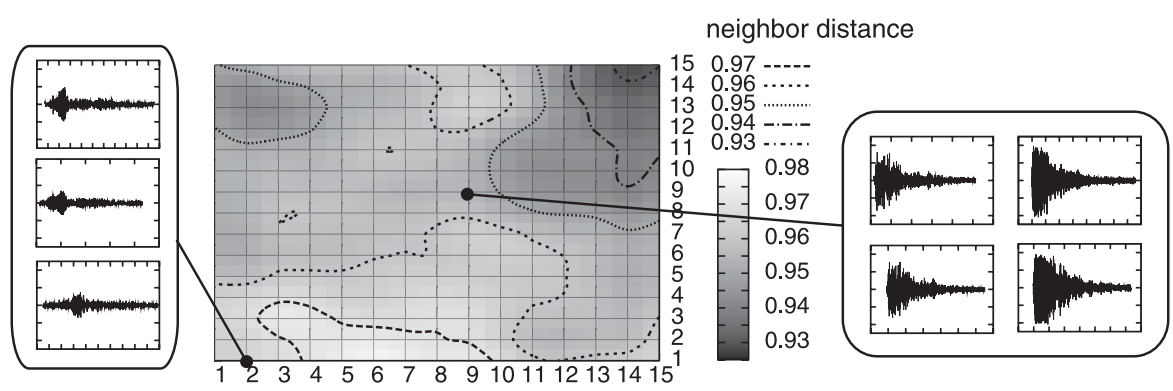

Fig. 9 Cluster map obtained by the kernel SOM.

Figure 8 shows an example of an extracted AE event and its preprocessed frequency spectrum. Moreover, each spectrum is normalized as $\Sigma_{k=1}^{v} x_{k}=1$ for the requirement of the KL kernel.

\section{Experimental Results}

\subsection{Visualization result by kernel SOM}

The learning results of the kernel SOM are shown in Fig. 9. The number of neurons was set to $15 \times 15$ with a two-dimensional regular grid. In general, the number of neurons is not sensitive to these results in the sense that the SOM captures the data distribution in the feature space. A grid point in the figure corresponds to a neuron, and similar AE events are clustered into the same or neighbor neuron. Note that the coordinates on the cluster map do not express any physical quantity but rather relative distances to the neighbor data.

In addition, the distance or dissimilarity between the neurons (grid points) is indicated by gray scale and contour by a U-matrix representation ${ }^{(22)}$. The smaller the value, the more closely the neurons are arranged, i.e., AE events on the cluster map are dense, and vice versa. The kernel parameter $\beta=0.95$ was determined by this inter-cluster distance among $\beta \in\{0.05,0.10, \cdots, 1.00\}$ that provides the clearest inter-cluster distance of the U-matrix representation.

\subsection{Time series analysis}

The damage process can be inferred from the temporal change in the regions of the cluster map in which AE events occur frequently (Fig. 10). The number of AE events allocated to the prototype neuron is indicated by the bar graph. Events that occurred for less than three times are not indicated in order to capture a comprehensive trend. Here, $n$ is not an actual time, but rather an AE event count, the correspondence to time of which is shown in Fig. 4. The events occurred during the heat up period until $n=113$ is indicated as one period. After $n=114$, each period consists of 200 successive counts, which overlap by 100 counts, so that the occurrence trend changes smoothly.

The regions in which AE events occur frequently change dynamically according to time in Fig. 10. The approximate regions and samples of AE events are illustrated in Fig. 11. Such regions imply a certain damage type. A physical interpretation of the cluster map was inferred by referring to actual $\mathrm{AE}$ waves, frequency spectrum within frequent occurring regions of the map, also to the temperature that those AE events occurred. In this manner, the damage type and phase transition were inferred as follows:

- Phase 1 (n=1-113) The AE events during this period occur intensively in region (a), as shown in Fig. 11, which implies that these AE events differ considerably from other events. Since this period is a moderate heat-up period, the cells are stable at this stage. Hence, the occurrence frequency remains low, and these continuous AE events with low energy are inferred as creaking of the members.

- Phase 2 ( $\mathbf{n = 1 1 4 - 5 0 0 )}$ The AE events during this period appear in region (b). In particular, the AE events during $\mathrm{n}=201-400$ appear around $(10,10)$, which is denoted as (b-1), and those during $n=201-600$ appear around $(15,15)$, which is denoted as (b-2). Region (b-1) 


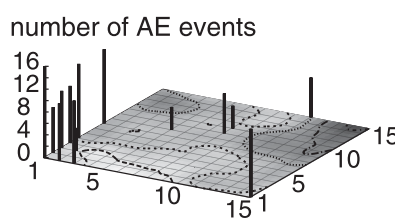

$\mathrm{n}=1-113$

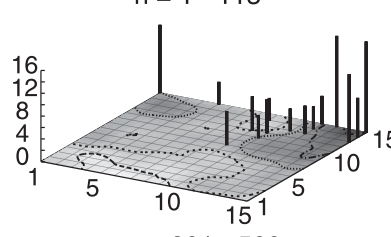

$\mathrm{n}=301-500$

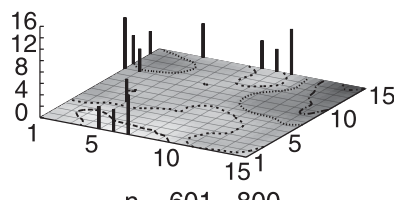

$n=601-800$

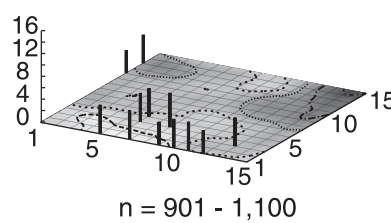

$n=901-1,100$

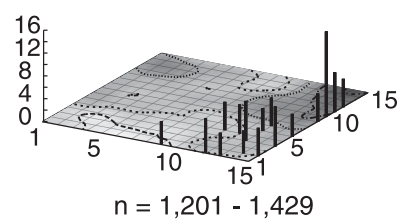

Fig. 10 Change in event count of AE events over time upon the cluster map by the kernel SOM.
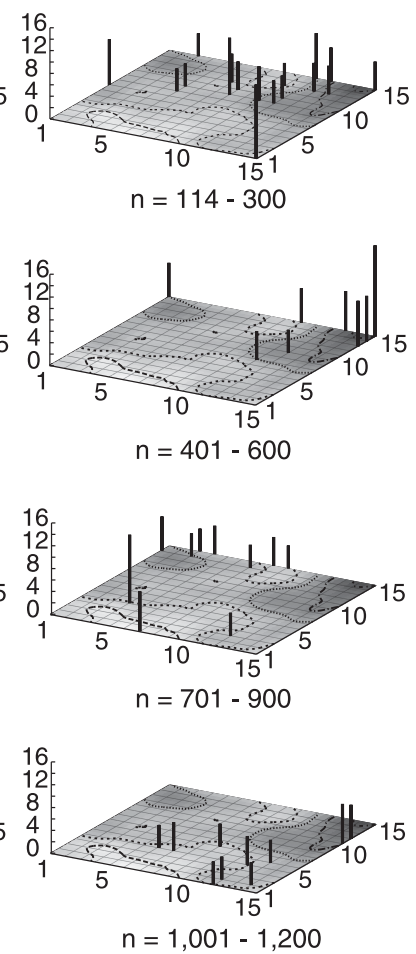
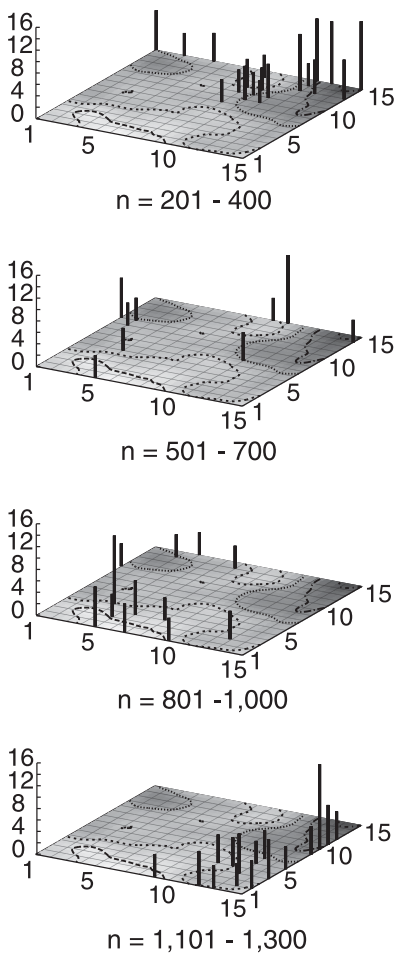

appears from the beginning of the temperature decrease with the outbreak type of high-energy $\mathrm{AE}$ events. Therefore, the events occurring in region (b-1) are inferred to be associated with the progression of the initial cracks, or other cracks, because of the unevenness of the materials at the beginning of constriction of the materials. Region (b-2) consists of continuous $\mathrm{AE}$ events with frequency spectra that are similar to the AE events in region (b-1). Therefore, region (b-2) is inferred as creaking of the members followed by region (b-1). Note that the similarity between spectra is measured based not on the absolute amplitude, but on the relative occurrence distribution.

- Phase 3 (n=501-800) Although there is no distinguishing frequent occurring region in this period, $\mathrm{AE}$ events in regions (c) and (d) relatively appear compared to other phases. The AE events in region (c) contain high-frequency spectra, and the temperature decreased to approximately $200^{\circ} \mathrm{C}$. Thus, the $\mathrm{AE}$ events in region (c) are thought to be associated with cracking of the electrolyte of a hard material because of stress caused by the solidification of the glass seal and constriction of the electrolyte. In region (d) of the cluster map, in which AE events occur frequently, shifts from left to right. The AE events in region (d) are thought to associated with damage to the glass seal. Since the glass seal is the only material for which the state of matter changes depending on the temperature, corresponding $\mathrm{AE}$ events change gradually as the temperature increases.

- Phase $4(n=801-1,429)$ In addition to damage to the glass seal of region (d), damage to region (e) frequently appears starting from $\mathrm{n}=1,101$ or later. The $\mathrm{AE}$ events in region (e) are a mixture of outbreak and continuous AE type damages with relatively low frequency spectra 


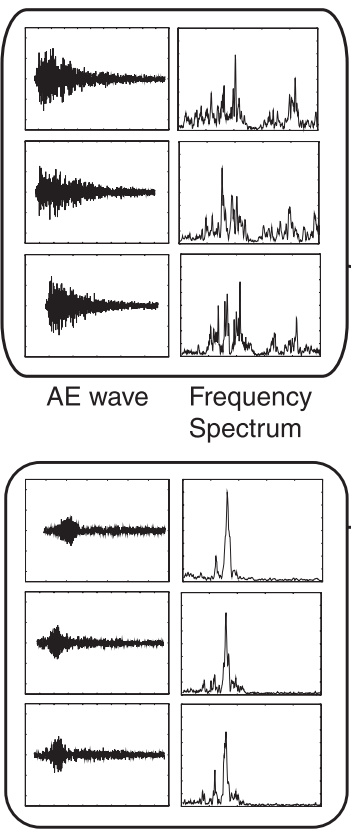

(a) Creaking of the members during heating up period

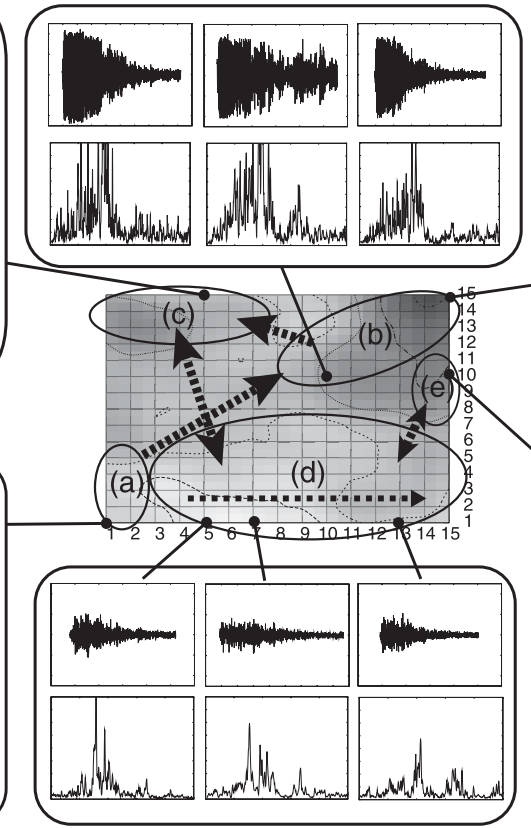

(d) Damages in the glass sea with changing its state

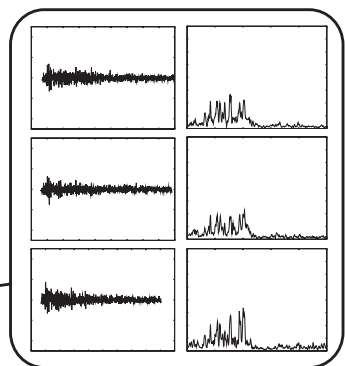

Fig. 11 Transition of frequent occurrence regions upon the cluster map and samples of each region.

of less than $50 \mathrm{kHz}$. Therefore, the AE events in region (e) are thought to be associated with cracks in the electrode and delamination between the electrode and the electrolyte.

\subsection{Future Perspective}

In the present study, we analyzed the progress of damage to the SOFC based on the occurrence trend of similar AE events. We succeeded in forming a hypothesis of the correspondence between $\mathrm{AE}$ events and actual damage types. The reliability of these hypotheses will be ensured by confirming reproducibility and/or physical simulation, as in Reference (23).

The automatic generation of a map that represents the similarity between AE events will help users to understand the phenomenon when a large number of various types of $\mathrm{AE}$ events exist, because the progress of damage to the SOFC was clarified for the first time in the present study. Moreover, the approach of the present study can be used to monitor SOFC operation. By constructing an SOFC damage database, the map can be made to show both the current status and future process of damage.

In the future, in addition to the frequency spectrum, other time-domain features such as duration, energy, and RA value (rise time/maximum amplitude), are candidates for improving the cluster map by extending the kernel SOM. In addition, in the present study, the occurrence trend of similar AE events was indicated by a sliding window method. We are planning to introduce the statistical inference of active regions of the cluster map that provide highresolution temporal change. Moreover, the extraction of mutual mechanical effects among materials based on the present research is expected.

\section{Conclusion}

In the present study, an approach by which to explore numerous acoustic emission events using the kernel Self-Organizing Map was developed as a basis for exploratory analysis, where the Kullback-Leibler divergence was introduced as an appropriate similarity for the frequency spectra of AE events. We demonstrated that the proposed approach can clarify the progress 
of damage to the SOFC based on the AE event sequence observed through a damage test. Regions of the cluster map obtained by the kernel SOM in which AE events occur frequently changed dynamically according to the progress of damage and are separated approximately into four phases. The proposed approach provides a common foundation for users to clarify the fracture mechanism of the SOFC and/or to monitor SOFC operation.

\section{Acknowledgements}

The present study was supported in part by a Management Expenses Grants for National Universities Corporations and by Grants-in-Aid for Scientific Research (21700165) through the Ministry of Education, Culture, Sports, Science and Technology of Japan (MEXT).

\section{References}

( 1 ) Singhal, S. C., Solid Oxide Fuel Cells for Stationary, Mobile, and Military Applications, Solid State Ionics, Vol. 152-153, (2002), pp. 405-410.

( 2 ) Yasuda, I. and Hishinuma, M., Lattice expansion of acceptor-doped lanthanum chromites under high-temperature reducing atmospheres, ELECTROCHEMISTRY, Vol. 68, No. 6, (2000), pp. 526-530.

( 3 ) Atkinson, A. and Ramos, T. M. G. M., Chemically-induced stresses in ceramic oxygen ion-conducting membranes, Solid Sate Ionics, Vol. 129, (2000), pp. 259-269.

( 4 ) Krishnamurthy, R. and Sheldon, B. W., Stresses due to oxygen potential gradients in non-stoichiometric oxides, Journal of Acta Materialia, Vol. 52, (2004), pp. 1807-1822.

( 5 ) Sato, K., Omura, H., Hashida, T., Yashiro, K., Kawada, T., Mizusaki, J. and Yugami, H., Tracking the Onset of Damage Mechanism in Ceria-based Solid Oxide Fuel Cells under Simulated Operating Conditions, Journal of Testing and Evaluation, Vol. 34, No. 3, (2006), pp. 246-250.

( 6 ) Kohonen, T., Self-Organizing Maps, (1995), Springer-Verlag.

( 7 ) Kohonen, T., Kaski, S., Lagus, K., Salojarvi, J., Honkela, J., Paatero, C. and Sarela, A., Self-Organization of a Massive Document Collection, IEEE Transactions on Neural Network, Vol. 11, No. 3, (2000), pp. 574-585.

( 8 ) Simula, O. and Kangas, J., Process monitoring and visualisation using Self-Organizing Maps, Neural Networks for Chemical Engineers, (1995), Elsevier Science B. V., pp. 377-390.

( 9 ) Moreno, P. J., Ho, P. P. and Vasconcelos, N, A Kullback-Leibler Divergence Based Kernel for SVM Classification in Multimedia Applications, Advances in Neural Information Processing Systems (NIPS), Vol. 16, (2003), pp. 1385-1392.

(10) Andras, P., Kernel-Kohonen networks, International Journal of Neural Systems, Vol. 12, No. 2, (2002), pp. 117-135.

(11) Boulet, R., Jouve, B., Rossi, F. and Villa, N., Batch Kernel SOM and Related Laplacian Methods for Social Network Analysis, Neurocomputing, Vol. 71, Issues 7-9, (2008), pp. 1257-1273.

(12) Miller, R. K., and Kill, E. V. K., Moore, P. O. and Hill, E. V., Acoustic Emission Testing, (2005), American Society for Nondestructive.

(13) Rippengill, S., Worden, K., Holford, K. M. and Pullin, R., Automatic Classification of Acoustic Emission Patterns, Journal for Experimental Mechanics: Strain, Vol. 39, No. 1, (2003), pp. 31-41.

(14) Godin, N., Huguet, S. and Gaertner, R., Influence of hydrolytic ageing on the acoustic emission signatures of damage mechanisms occurring during tensile tests on a polyester composite: Application of a Kohonen's map, Composite Structures, Vol. 72, No. 1, (2006), pp. 79-85.

(15) Fukui, K., Sato, K., Mizusaki, J., Saito, K. and Numao, M., Combining Burst Extraction Method and Sequence-based SOM for Evaluation of Fracture Dynamics in Solid Oxide 
Fuel Cell, Proc. 19th IEEE International Conference on Tools with Artificial Intelligence (ICTAI-07), Vol. 2, (2007), pp. 193-196.

(16) Fukui, K., Sato, K., Mizusaki, J. and Numao, M, Kullback-Leibler Divergence Based Kernel SOM for Visualization of Damage Process on Fuel Cells, Proc. of 22th IEEE International Conference on Tools with Artificial Intelligence (ICTAI-10), Vol. 1, (2010), pp. 233-240.

(17) Bishop, C. M., Pattern Recognition and Machine Learning, (2006), Springer-Verlag.

(18) Cristianini, N. and Shawe-Taylor, J., An Introduction to Support Vector Machines and Other Kernel-Based Learning Methods (2000), Cambridge University Press.

(19) Ishigaki, T. and Higuchi, T., Dynamic Spectrum Classification by Kernel Classifiers with Divergence-Based Kernels and its Applications to Acoustic Signals International Journal of Knowledge Engineering and Soft Data Paradigms, Vol. 1, No. 2, (2009), pp. 173-192.

(20) Kleinberg, J., Bursty and Hierarchical Structure in Streams, Data Mining and Knowledge Discovery, Vol. 7, No. 4, (2003), pp. 373-397.

(21) Viterbi, A. J., Error Bounds for Convolutional Codes and an Asymptotically Optimum Decoding Algorithm, IEEE Transactions on Information Theory, Vol. 13, No. 2, (1967), pp. 260-269.

(22) Ultsch, A. and Siemon, H. P., Self-Organizing Feature Maps for Exploratory Data Analysis, Proc. the International Neural Network Conference (INNC), (1990), pp. 305-308.

(23) Sato, K., Yashiro, K., Kawada, T., Yugami, H., Hashida, T. and Mizusaki, J., Fracture Process of Nonstoichiometric Oxide Based Solid Oxide Fuel Cell under Oxidizing/Reducing Gradient Conditions, Journal of Power Sources, Vol. 195, Issue 17, (2010), pp. 5481-5486. 